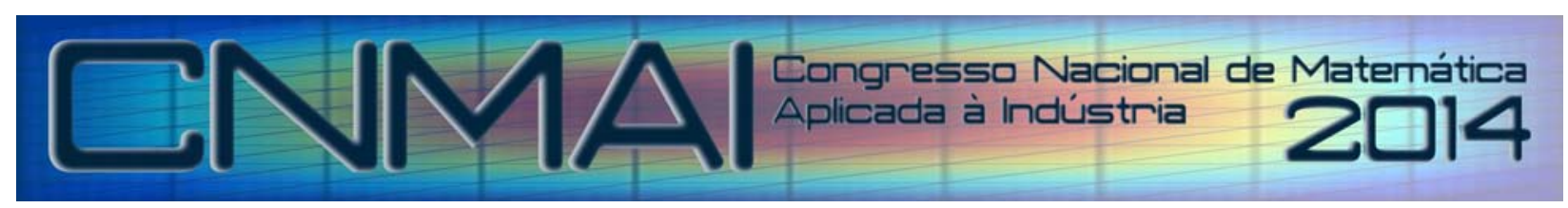

18 a 21 de novembro de 2014, Caldas Novas - Goiás

\title{
COMPARATIVO ENTRE MODELOS MATEMÁTICOS DE TURBINA EÓLICA DE EFEITO MAGNUS E CONVENCIONAL
}

\author{
Maro Jinbo, marojinbo@gmail.com ${ }^{1,3}$ \\ Felix Alberto Farret, fafarret@gmail.com ${ }^{1}$ \\ Ghendy Cardoso Junior, ghendy@ufsm.br ${ }^{1}$ \\ Edson Ribeiro dos Santos, edson.santos@uffs.edu.br ${ }^{2}$ \\ Daniel Senter, danielsenter1@gmail.com ${ }^{3}$ \\ Jawilson Pereira Machado, e-mail: machado@uceff.edu.br ${ }^{4}$ \\ ${ }^{1}$ Universidade Federal de Santa Maria, UFSM - Departamento de Engenharia Elétrica. \\ Avenida Roraima, 1000 Santa Maria/RS. \\ ${ }^{2}$ Universidade Federal da Fronteira Sul, UFFS - Departamento de Matemática. \\ Avenida Fernando Machado, 108 Chapecó/SC. \\ ${ }^{3}$ Instituto Federal de Santa Catarina, IFSC - Departamento de Eng. Controle e Automação. \\ Avenida Nereu Ramos, 3450-D Chapecó/SC. \\ ${ }^{4}$ Faculdade Empresarial de Chapecó (UCEFF) - Departamento de Engenharia Ambiental e Sanitária. \\ Rua Lauro Muller, 767 E, Chapecó/SC.
}

Resumo: Este artigo apresenta a modelagem matemática de turbinas eólicas que não utilizam pás convencionais para captar a energia cinética dos ventos. No lugar das pás, a turbina possui cilindros girantes acionados por um ou mais motores elétricos. Cilindros girantes imersos no ar criam diferenças de pressões originando forças (de sustentação) transversais à direção do fluxo de ar. Este fenômeno físico é conhecido como Efeito Magnus. Essas forças aplicadas aos cilindros girantes de uma turbina proporcionam o torque no eixo principal que é transmitida ao gerador elétrico. Apresenta-se o modelo matemático de turbinas eólicas convencionais e realiza-se um comparativo em termos de potência mecânica com a turbina eólica Magnus.

Palavras-chave: Turbina Eólica; Efeito Magnus; Cilindros Girantes; Turbinas de baixa velocidade.

\section{INTRODUÇÃO}

$\mathrm{Na}$ Figura 1 tem-se um cilindro de raio $\mathrm{R}$ rotacionando com velocidade angular $\Omega$, imerso em um fluido de densidade $\rho$, cuja velocidade laminar do fluido é $U_{\infty}$. Teoricamente o cilindro está sujeito a uma força resultante na direção perpendicular ao fluxo do fluido denominado força de sustentação $\mathrm{F}_{\mathrm{Y}}$ (lift force) e de arrasto $\mathrm{F}_{\mathrm{X}}$ (drag force), fenômeno conhecido como Efeito Magnus (Munson, 2009; White, 2012). Em teoria a força de arrasto $F_{X}$ é nula, mas experimentalmente comprova-se que este valor não é nulo e aumenta com o aumento da velocidade relativa da superfície do cilindro (White, 2012). 


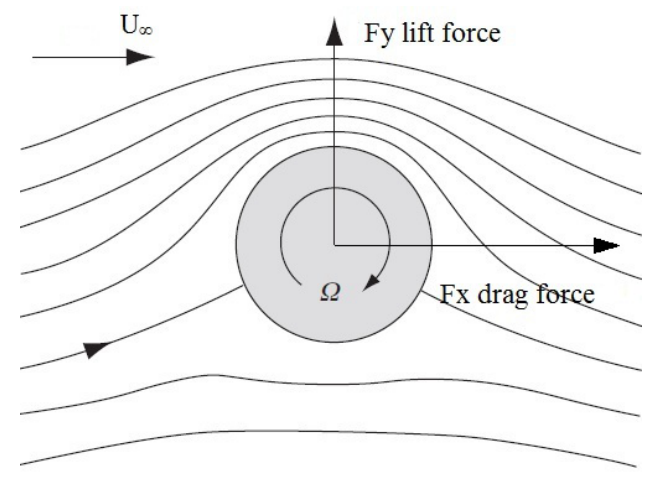

Figura 1 - Cilindro rotacionando imerso em um fluido (Burton, 2011).

A turbina eólica de efeito Magnus possui cilindros girantes no lugar das pás convencionais conforme Fig. 2. As velocidades angulares (rotação) $\Omega_{1}$ e $\Omega_{2}$ são da turbina e dos cilindros respectivamente. Seu funcionamento baseia-se no princípio físico conhecido por Efeito Magnus. A Figura 2 mostra o detalhe (a) que consiste na porção frontal do cubo do rotor onde está localizado o sistema de servo acionamento CC (servo drive CC e o motor brushless CC). A transmissão da rotação do motor brushless $\mathrm{CC}$ para os cilindros, detalhe (b), é realizado por um sistema de engrenagens.

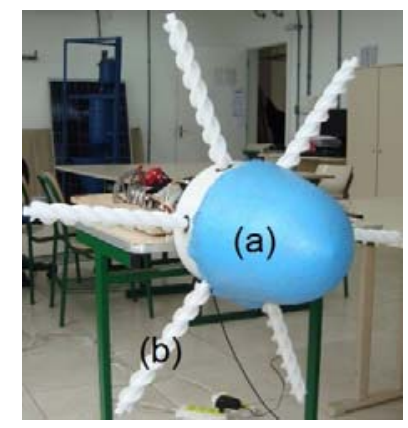

Figura 2 - Turbina eólica de efeito Magnus (IFSC/UFSM, 2013): (a) Cubo. (b) Cilindro.

Não são recentes os registros dos primeiros protótipos de turbinas eólicas de efeito Magnus. Mais recentemente uma empresa japonesa através de esforços em conjunto entre indústria, governo e universidades desenvolveram e lançaram comercialmente uma turbina eólica de efeito Magnus. Os cilindros desta turbina Magnus possuem duas características relevantes, uma barbatana em espiral e uma tampa na extremidade (Murakami, 2009).

$\mathrm{Na}$ Rússia realizaram-se experimentos com protótipos de turbinas eólicas Magnus em um túnel de vento. Comprovou-se que a turbina Magnus mesmo em velocidades de vento de $2 \mathrm{~m} / \mathrm{s}$ apresenta um coeficiente de potência suficiente para gerar potência útil e aproveitar estas baixas velocidades de vento. A turbina pode ser operada também para velocidades maiores que 35 a $40 \mathrm{~m} / \mathrm{s}$ de vento, bem superiores ao limite das turbinas convencionais (Bychkov, 2007; Bychkov e Dovga; 2008; Bychkov, 2008).

\section{TURBINA EÓLICA DE EFEITO MAGNUS}

\subsection{A Potência da Turbina Eólica Magnus}

A Figura 3 apresenta uma turbina eólica de efeito Magnus, onde:

$\mathrm{N}$ = número de cilindros girantes;

$\mathrm{R}_{1}=$ raio da turbina $[\mathrm{m}]$;

$\Omega_{1}=$ velocidade angular da turbina $[\mathrm{rad} / \mathrm{s}]$;

$\mathrm{R}_{2}=$ raio dos cilindros [m];

$\Omega_{2}=$ velocidade angular dos cilindros $[\mathrm{rad} / \mathrm{s}] ;$

$\mathrm{U}_{\infty}=$ velocidade laminar do vento $[\mathrm{m} / \mathrm{s}]$;

$\rho=$ densidade do ar $\left[\mathrm{kg} / \mathrm{m}^{3}\right]$.

A razão entre a velocidade tangencial da extremidade do cilindro da turbina e a velocidade do vento é conhecida como TSR ( Tip Speed Ratio) da turbina Magnus, (Slootweg, 2003). A TSR do rotor é dada pela Eq. (1) : 


$$
\lambda_{1}=\frac{\Omega_{1} \cdot R_{1}}{U_{\infty}}
$$

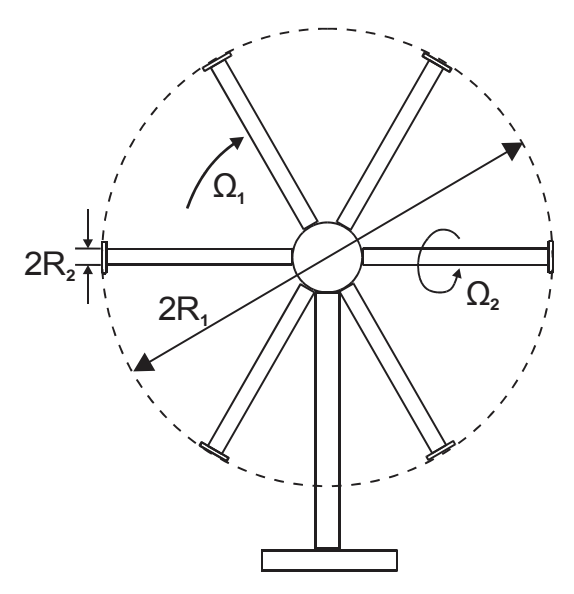

Figura 3 - Turbina eólica de efeito Magnus.

A expressão da velocidade relativa (TST) do cilindro em relação à velocidade do vento é definida pela Eq. (2) (Luo, 2011; Sun, 2012):

$$
\lambda_{2}=\frac{\Omega_{2} \cdot R_{2}}{U_{\infty}}
$$

Portanto, a potência mecânica extraída do vento pela turbina Magnus é representada pela Eq. (3) (Burton, 2011; Slootweg, 2003; Luo, 2011; Sun, 2012; Monroy, 2006):

$$
P=0,5 \cdot \rho \cdot U_{\infty}^{3} \cdot \pi \cdot R_{1}^{2} \cdot C_{P}
$$

A Equação (4) analítica é referente ao coeficiente de potência $C_{P}$ da turbina de efeito Magnus e foi obtida com base nos princípios da dinâmica dos fluidos e da teoria da quantidade de movimento do elemento pá (BEM - Blade Element Momentum Theory) desprezando-se a influência de outros fatores relevantes como força de arrasto, perdas nas extremidades dos cilindros, interação entre os cilindros e o efeito do diâmetro do cubo na potência de saída da turbina. $\mathrm{O}$ modelo é melhorado com valores de $\mathrm{C}_{\mathrm{P}}$ obtidos experimentalmente que representa mais proximamente a realidade (Luo, 2011; Sun, 2012). O coeficiente de potência representa o percentual de potência que a turbina consegue extrair dos ventos. $\mathrm{O}$ valor limite do coeficiente de potência para turbinas eólicas de efeito Magnus é $\mathrm{C}_{\mathrm{P}}=0.593$.

$$
C_{P}=\frac{N \cdot \Omega_{1} \cdot \Omega_{2} \cdot R_{2}^{2}}{2 \cdot U_{\infty}^{2}} \cdot\left(1+\sqrt{1-\frac{N \cdot \Omega_{1} \cdot \Omega_{2} \cdot R_{2}^{2}}{U_{\infty}^{2}}}\right)
$$

\section{MODELO DINÂMICO DAS TURBINAS EÓLICAS CONVENCIONAIS}

Os fabricantes de turbinas eólicas convencionais de eixo horizontal não costumam disponibilizar prontamente as informações sobre o coeficiente de potência de seus produtos. Existem várias aproximações numéricas desenvolvidas

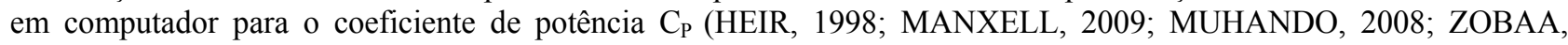
2011). A Equação (5) juntamente com Eq. (6) é uma aproximação de uma função não linear para as turbinas eólicas convencionais de 3 pás.

$$
\mathrm{C}_{\mathrm{P}}(\lambda, \beta)=\mathrm{C}_{1}\left(\mathrm{C}_{2} \lambda_{\mathrm{i}}-\mathrm{C}_{3} \beta-\mathrm{C}_{4}\right) \mathrm{e}^{-\mathrm{C}_{5} \lambda_{\mathrm{i}}}+\mathrm{C}_{6} \lambda
$$




$$
\lambda_{\mathrm{i}}=\left(\frac{1}{\lambda+0.08 \beta}-\frac{0.035}{\beta^{3}+1}\right)
$$

Na Tabela 1 constam os valores dos coeficientes $\mathrm{C}_{1}, \mathrm{C}_{2}, \mathrm{C}_{3}, \mathrm{C}_{4}, \mathrm{C}_{5}$ e $\mathrm{C}_{6}$ para uma turbina eólica convencional com 3 pás (SONG,2003), sendo que o $\beta$ é o ângulo das pás em relação à direção do vento:

Tabela 1 - Coeficientes de $\mathrm{C}_{\mathrm{P}}$ de turbine eólica convencional de 3 pás.

\begin{tabular}{c|c|c|c|c|c}
\hline $\mathrm{C}_{1}$ & $\mathrm{C}_{2}$ & $\mathrm{C}_{3}$ & $\mathrm{C}_{4}$ & $\mathrm{C}_{5}$ & $\mathrm{C}_{6}$ \\
\hline 0.5176 & 116 & 0.4 & 5 & 21 & 0.0068 \\
\hline
\end{tabular}

A Equação (7) é a relação entre a velocidade tangencial da extremidade das pás e a velocidade do vento conhecido como TSR - Tip Speed Ratio similar ao da turbina Magnus:

$$
\lambda=\frac{\omega_{\mathrm{t}} \cdot \mathrm{R}}{\mathrm{U}_{\infty}}
$$

O coeficiente de potência $C_{P}$ varia em função de $\lambda$ (TSR), Eq. (7), onde $\omega_{t}$ é a velocidade de rotação da turbina em $\mathrm{rad} / \mathrm{s}$ e $U_{\infty}$ a velocidade do vento laminar. $O \mathrm{C}_{\mathrm{P}}$ representa o desempenho da turbina, ou seja, o quanto de potência ele consegue capturar do vento.

Na Figura 4 apresenta-se a curva do coeficiente de potência $C_{P}$ em função da velocidade relativa da turbina $\lambda$ para as turbinas modernas de 3 pás. $O$ máximo valor de $C_{P}$ é apenas 0.47 com valor de $\lambda$ igual a 7 , o que consiste em um valor menor que o limite de Betz, devido às perdas por arrasto, turbulências nas extremidades das pás e perdas por stall (BURTON et. al., 2011).

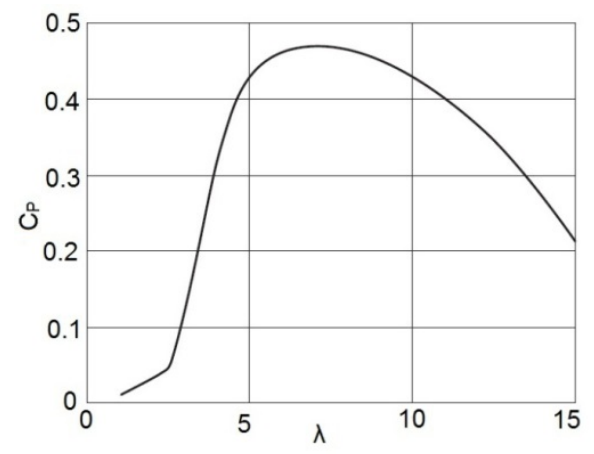

Figura $4-\mathrm{C}_{\mathrm{P}}$ em função de $\lambda$ (TSR) de modernas turbinas de 3 pás Fonte: Burton et. al., 2011.

As curvas de $\mathrm{C}_{\mathrm{P}}$ apresentadas na Figura 5 foram obtidas com a Eq. (5) utilizando-se os coeficientes da Tabela 1 referentes às turbinas eólicas horizontais convencionais de 3 pás.

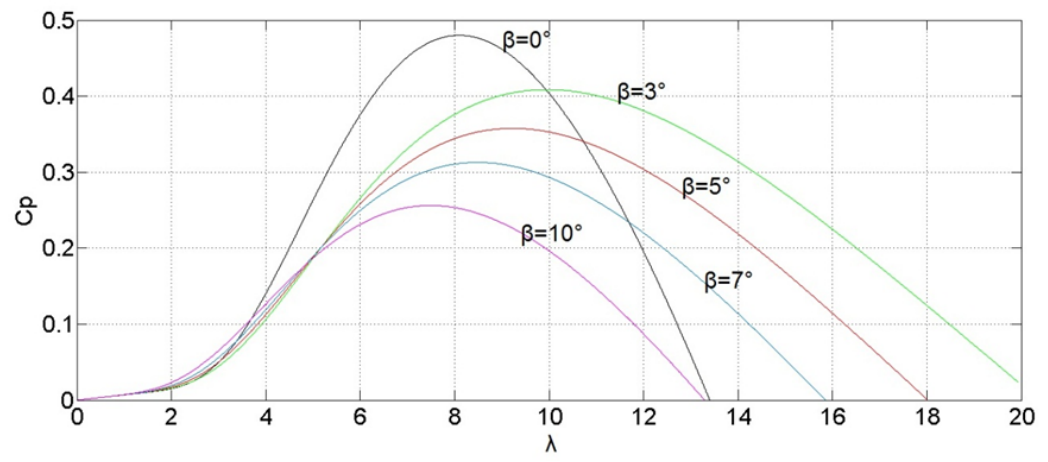

Figura 5 - Variação de $C_{P} \operatorname{com} \beta$ e $\lambda$ de turbinas eólicas de 3 pás

\section{RESULTADOS DE SIMULAÇÃO}

Nesta seção apresentam-se as curvas de potências mecânicas teóricas produzidas pelas turbinas eólicas convencional e Magnus de acordo com a Eq. (3) para várias velocidades de ventos possibilitando um comparativo. As potências 
mecânicas geradas pelas turbinas dependem do seu coeficiente de potência $C_{P} . O C_{P}$ da turbina convencional é de acordo com a Eq. (5) e o $\mathrm{C}_{\mathrm{P}}$ da turbina Magnus de acordo com a Eq. (4). As curvas de potência da turbina convencional nas figuras desta seção estão em função do TSR e as curvas de potência da turbina Magnus estão em função de $\lambda_{2}$ (velocidade relativa dos cilindros girantes). Estas curvas não levam em consideração a potência elétrica necessária para o acionamento elétrico dos cilindros girantes no caso das figuras de potência da turbina Magnus.

Através de medições realizadas em um protótipo construído de turbina Magnus de poliacetal com 6 cilindros de 35 $\mathrm{cm}$ de comprimento e $4 \mathrm{~cm}$ de diâmetro (IFSC/UFSM) conforme Fig. 2, pode-se considerar que a potência elétrica consumida pelo motor de acionamento dos cilindros fique em torno de $37.5 \mathrm{~W}(25 \mathrm{~V} 1.5 \mathrm{~A})$. Portanto, das curvas de potência mecânica da turbina Magnus das figuras desta seção pode-se subtrair este valor de potência consumida para realizar uma comparação mais precisa.

Tabela 2 - Parâmetros da Turbina Magnus $\mathrm{R}_{1}=1.15 \mathrm{~m}$ e $\mathrm{R}_{2}=0.025 \mathrm{~m}$.

\begin{tabular}{ccc}
\hline Parâmetro & Descrição & Valor \\
\hline $\mathrm{N}$ & Número de cilindros & 6 \\
\hline $\mathrm{R}_{1}[\mathrm{~m}]$ & Raio da turbina & 1.15 \\
\hline $\mathrm{R}_{2}[\mathrm{~m}]$ & Raio dos cilindros & 0.025 \\
\hline Vento $1[\mathrm{~m} / \mathrm{s}]$ & Faixa de maior incidência & 3.5 a 7.5 \\
\hline Vento $2[\mathrm{~m} / \mathrm{s}]$ & Faixa de baixa incidênica & 7.5 a 12.5 \\
\hline Vento $3[\mathrm{~m} / \mathrm{s}]$ & Faixa de rara incidência & 12.5 a 40 \\
\hline$\lambda_{1}$ & TSR Tip Speed Ratio & 1.0 \\
\hline$\Omega_{1}[\mathrm{RPM}]$ & Rotação da turbina & 0 a 48 \\
\hline$\lambda_{2}$ & Veloc. relativa do cilindro & 0 a 1 \\
\hline$\Omega_{2}[\mathrm{RPM}]$ & Rotação dos cilindros & 0 a 2865 \\
\hline
\end{tabular}

Na Figura 6(a) apresentam-se as curvas de potências teóricas geradas em função da velocidade relativa dos cilindros $\lambda_{2}$ para uma turbina Magnus conforme dados da Tab. 2. A faixa de velocidade do vento considerada na literatura de maior incidência. Na Figura 6(b) apresentam-se as curvas de potências teóricas geradas em função da velocidade relativa da turbina $\lambda_{1}$ (TSR) para uma turbina convencional de mesmo raio da turbina Magnus.

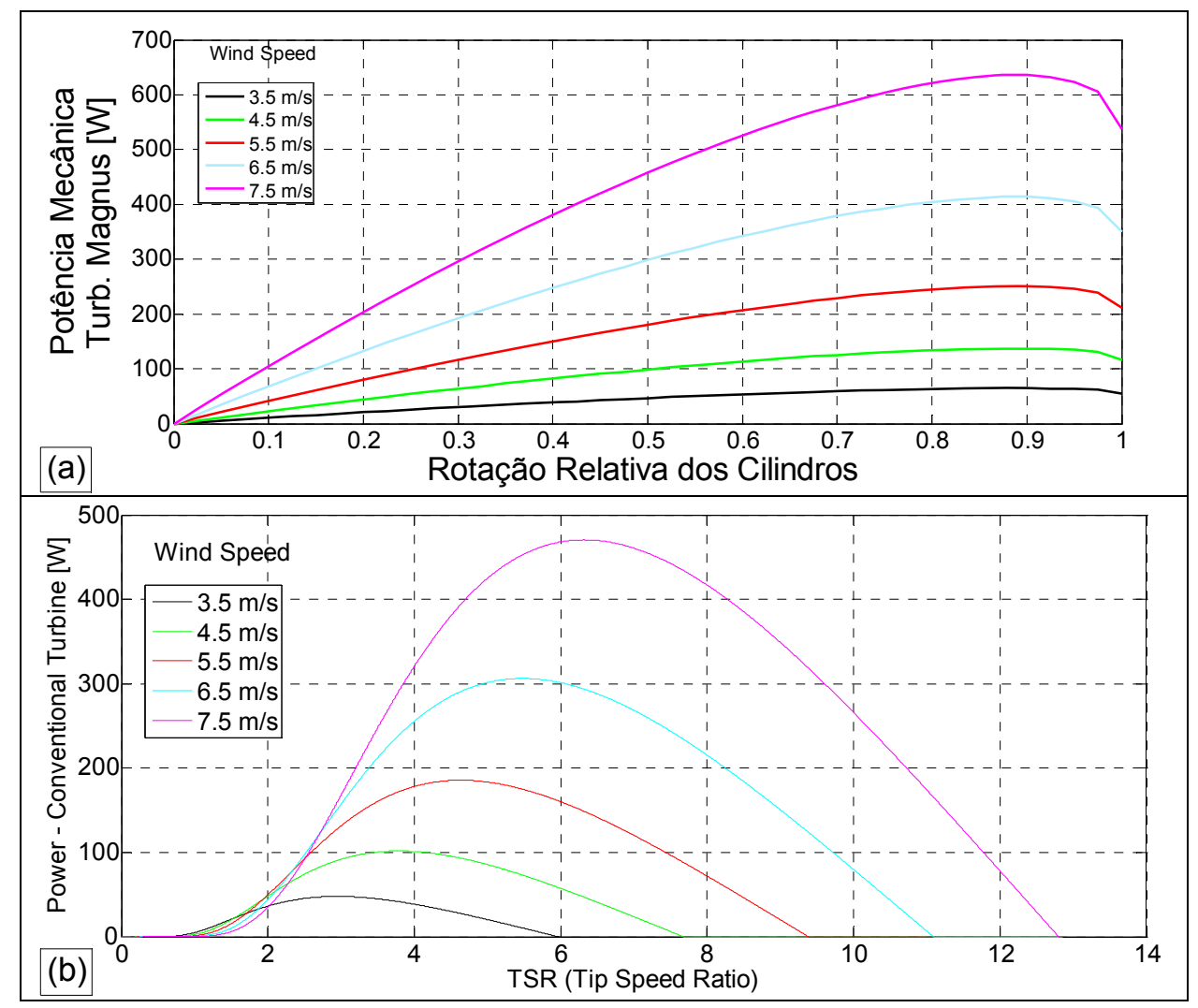

Figura 6 - Potências mecânicas calculadas para ventos de maiores incidências. a) Turbina Magnus. b) Turbina Convencional. 
Na Figura 7(a) apresentam-se as curvas de potências geradas calculadas em função da rotação relativa dos cilindros $\lambda_{2}$ para uma turbina Magnus conforme dados da Tab. 2. Na Figura 7(b) apresentam-se as curvas das potências teóricas geradas em função da velocidade relativa da turbina $\lambda_{1}$ (TSR) para uma turbina convencional de mesmo raio da turbina Magnus. A faixa de velocidades dos ventos é considerada de baixas incidências.

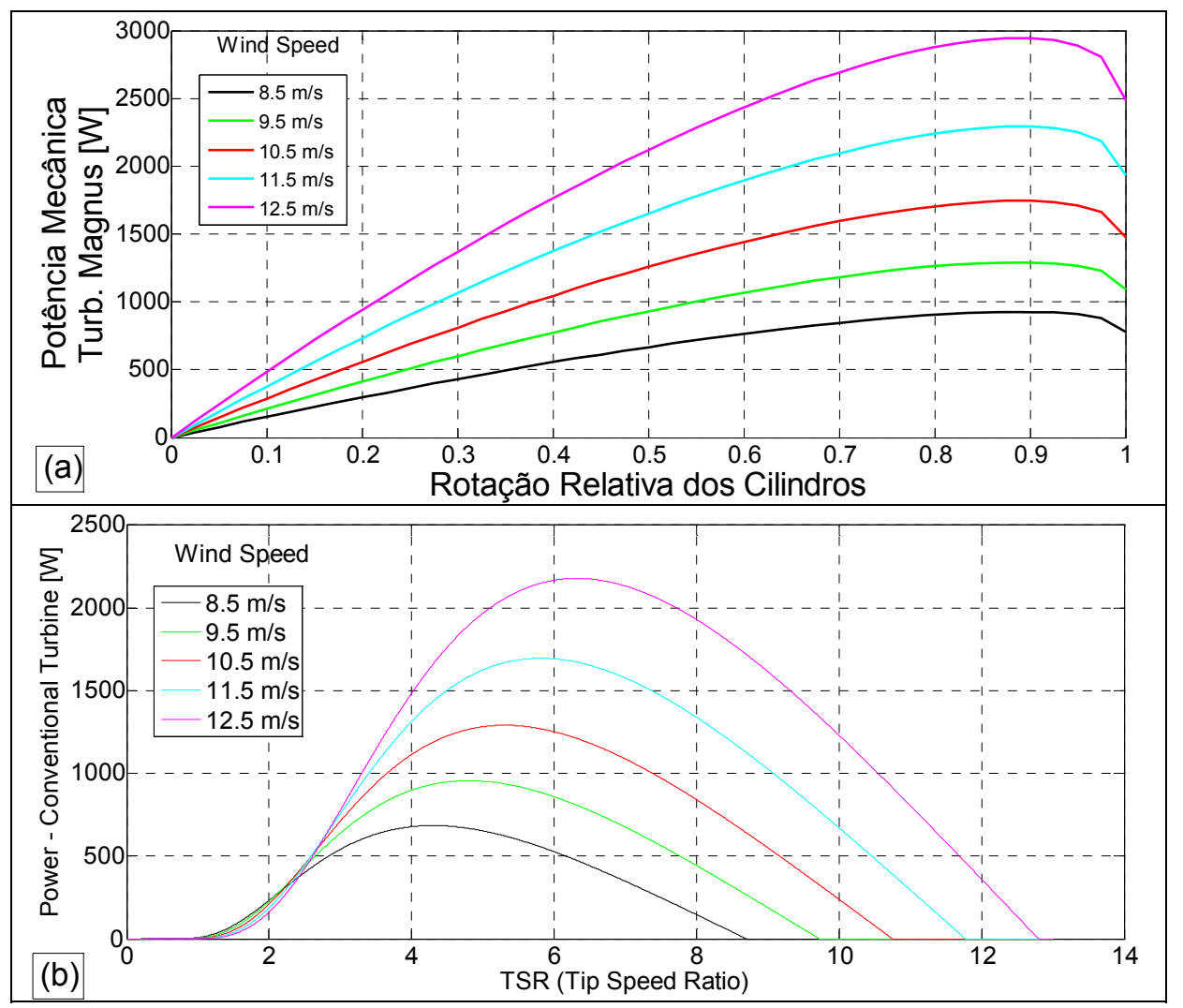

Figura 7 - Potências calculadas na faixa de ventos de baixas incidências.

a) Turbina Magnus b) Turbina Convencional

Na Figura 8(a) apresentam-se as curvas de potências calculadas em função da rotação relativa dos cilindros $\lambda_{2}$ para uma turbina Magnus conforme dados da Tab. 2. Na Figura 8(b) apresentam-se as curvas das potências teóricas geradas em função da velocidade relativa da turbina $\lambda_{1}$ (TSR) para uma turbina convencional de mesmo raio da turbina Magnus. A faixa de velocidades dos ventos de raras incidências.

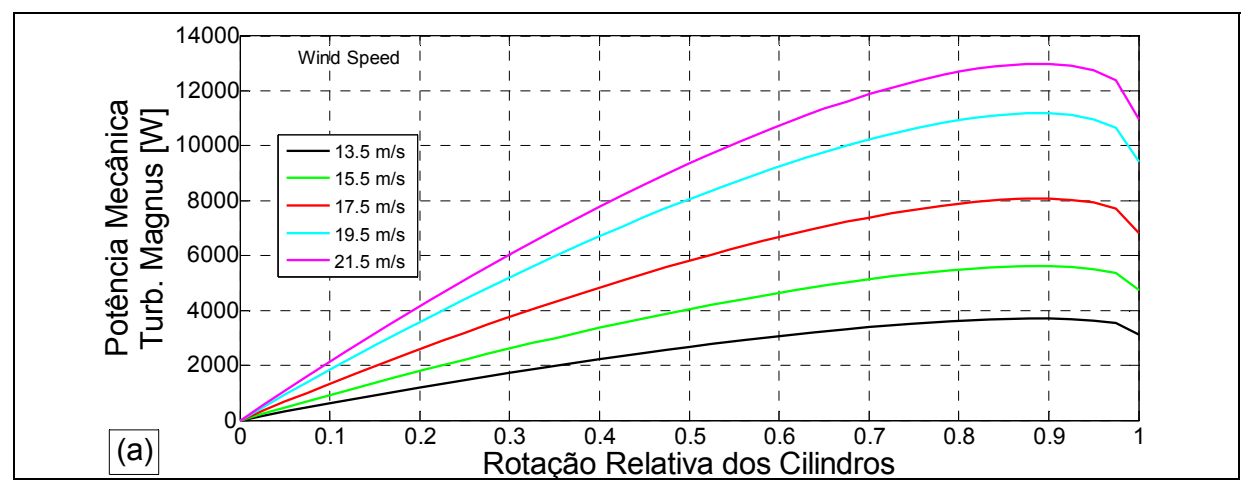




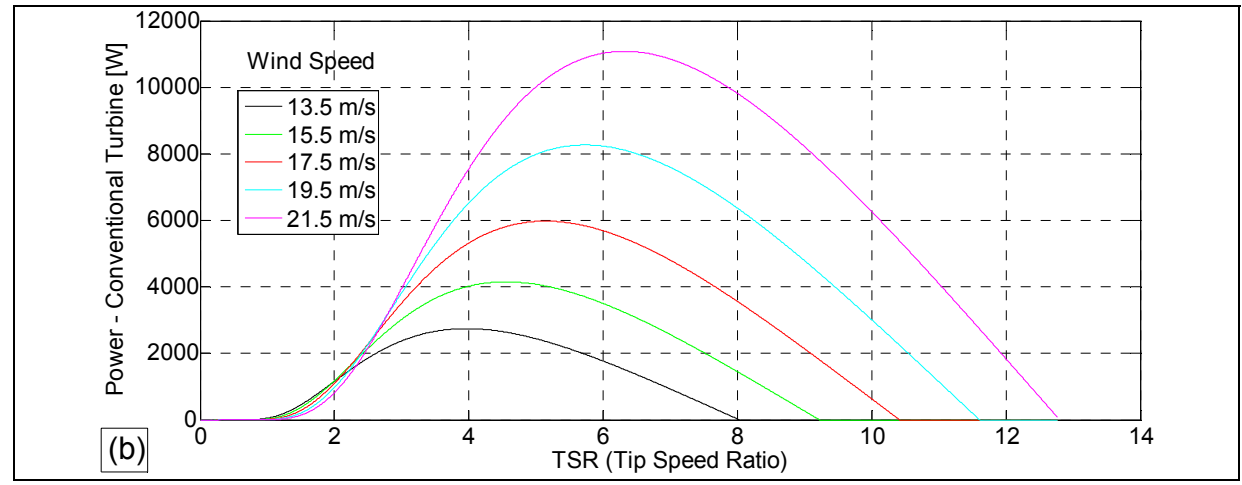

Figura 8 - Potências calculadas na faixa de ventos de baixas incidências. a) Turbina Magnus b) Turbina Convencional

A Figura 9 apresenta as curvas de potências mecânicas teóricas geradas turbinas Magnus para uma velocidade de vento de $7.5 \mathrm{~m} / \mathrm{s}$ para 5 comprimentos de cilindros diferentes com raio de $\mathrm{R}_{2}=0.025 \mathrm{~m}$.

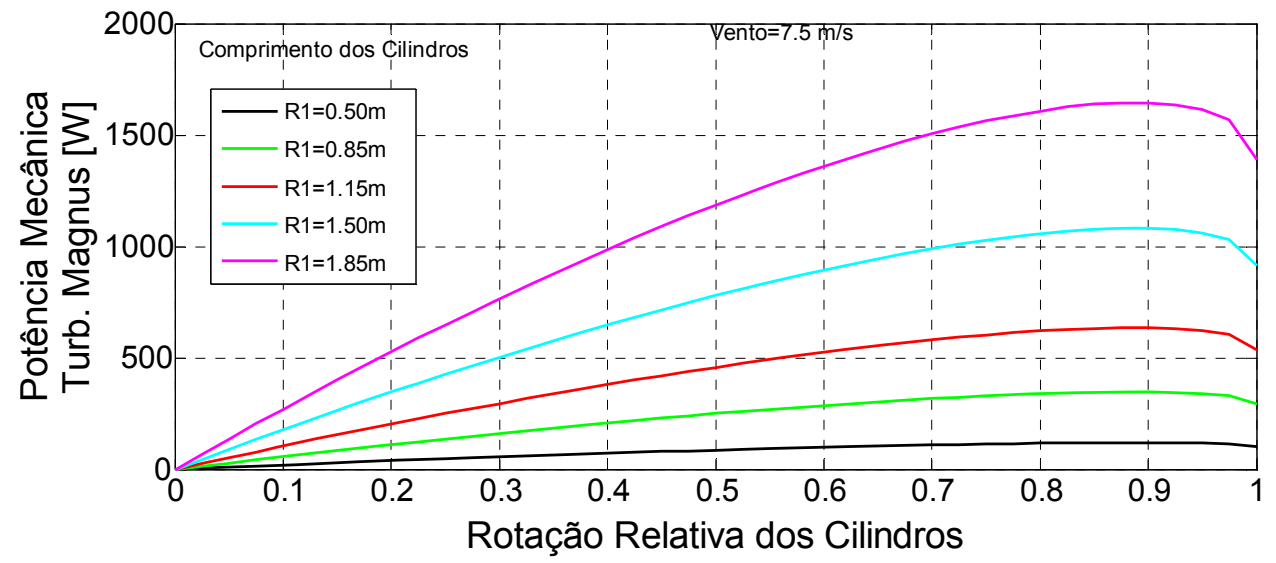

Figura 9 - Potência calculada para vento de $7.5 \mathrm{~m} / \mathrm{s}$.

Variações no diâmetro da turbina Magnus.

\section{CONCLUSÃO}

O modelo matemático do coeficiente de potência $\mathrm{C}_{\mathrm{P}}$ da turbina eólica de efeito Magnus melhorada com resultados experimentais em túnel de vento de LUO (2010) representada pela Eq. (4) foi aplicada na equação de potência mecânica de turbinas eólicas, Eq. (3). Com esta equação de potência obtida gerou-se as curvas de potência apresentadas de forma comparativa com as curvas de potência de uma turbina eólica convencional de 3 pás representadas pela Eq. (5). Nesta comparação entre potências teóricas geradas, levando-se em consideração a potência elétrica consumida por um motor elétrico sem escovas do tipo brushlesss pode-se destacar o desempenho teórico superior da turbina Magnus em relação às turbinas eólicas convencionais para este diâmetro de turbinas. Pode-se constatar que o aumento do comprimento dos cilindros implica no aumento da potência mecânica da turbina Magnus.

\section{ACKNOWLEDGEMENT}

The authors would like to thank CNPq and CEESP-CT-UFSM for the financial support given to the development of this work.

\section{REFERÊNCIAS}

www.ufsm.br/ppgee / www.ifsc.edu.br

Munson, B.R.; Young, D.F.; Okiishi, T.H.; Huebsch, W.W. "Fundamentals of Fluid Mechanics". 8 ed. John Wiley \& Sons, Inc. 2009. Pp. 304.

White, F.M. "Fluid Mechanics". 4 ed. McGraw Hill. 2012. Pp. 509-510; pp. 518.

Burton, T.; Jenkins, N.; Sharpe, D.; Bossanyi, E. "Wind Energy Handbook”. 2 ed. John Wiley \& Sons, 2011. pp. 4-5; pp. 127.

Murakami, N.; Ito, J.. “Magnus Type Wind Power Generator”. United States Patent. US7.504.740 B2. March, 
17, 2009.

Bychkov, N.; Dovgal, A.; Kozlov, V. "Magnus Wind Turbine as an Alternative to the Blade Ones". Journal of Physics: Conference Series 75, 2007.

Bychkov, N.; Dovgal, A.; Sorokin, A. "Parametric Optimization of the Magnus Wind Turbine". International Conference on Methods of Aerophysical Research, ICMAR 2008.

Bychkov, N.M. "Magnus Wind Turbine, Calculated Characteristics of the Wind Wheel". Thermophysics and Aeromechanics, Vol.15 no2, 2008.

Slootweg, J.G.; Haan, W.H.; Polinder, H.; Kling, W.L. "General Model for Representing Variable Speed Wind Turbines in Power System Dynamics Simulations". IEEE Trans. Power Systems, Vol.18, No.1, Feb. 2003.

Luo; D.; Huang, D; Wu, G. "Analytical solution on Magnus wind turbine power performance based on the blade element momentum theory". Journal of Renewable and Sustainable Energy 3, 2011.

Sun, X.; Zhuang, Y.; Cao, Y.; Huang, D.; Wu, G. "A three-dimensional numerical study of the Magnus wind turbine with different blade shapes". Journal of Renewable and Sustainable Energy 4, 2012.

Monroy, A.; Icaza, L.A. "Real-time identification of wind turbine rotor power coefficient." Proceedings of the 45th IEEE Conference on Decision \& Control, 2006.

Han, K; Chen, G. "A Novel Control Strategy of Wind Turbine MPPT Implementation for Direct-drive PMSG Wind Generation Imitation Platform". IEEE 2009.

Yang, X.; Gong, X.; Qiao,W. "Mechanical Sensorless Maximum Power Tracking Control for Direct-Drive PMSG Wind Turbines". IEEE 2010.

Duan, R., Lin, C.; Wai, R. "Maximum-Power-Extraction Algorithm for Grid-Connected PMSG Wind Generation System". IEEE 2006.

Ribeiro, C.G.; Farret, F.A; Corrêa, L.C.; Trapp, J.G.; Lenz, J.M. "Maximum Power Point Tracking for Magnus Wind Turbines". IEEE2013.

Simões, M.G.; Farret F.A. Renewable Energy Systems. Design and Analysis with Induction Generators. CRC Press, 2004. pp. 197-208.

\title{
RESPONSABILIDADE AUTORAL
}

Os autores são os únicos responsáveis pelo conteúdo deste trabalho

\section{COMPARISON BETWEEN MATHEMATICAL MODELS OF THE MAGNUS EFFECT WIND TURBINE AND CONVENTIONAL TURBINE}

\author{
Maro Jinbo, marojinbo@gmail.com ${ }^{1,3}$ \\ Felix Alberto Farret, fafarret@gmail.com ${ }^{1}$ \\ Ghendy Cardoso Junior, ghendy@ufsm.br ${ }^{1}$ \\ Edson Ribeiro dos Santos, edson.santos@uffs.edu.br ${ }^{2}$ \\ Daniel Senter, danielsenter1@gmail.com ${ }^{3}$ \\ Jawilson Pereira Machado, e-mail: machado@uceff.edu.br ${ }^{4}$ \\ ${ }^{1}$ Federal University of Santa Maria, UFSM - Department of Electrical Engineering. \\ Avenida Roraima, 1000 Santa Maria/RS. \\ ${ }^{2}$ Federal University of Fronteira Sul, UFFS - Department of Mathematic. \\ Avenida Fernando Machado, 108 Chapecó/SC. \\ ${ }^{3}$ Federal Institute of Santa Catarina, IFSC - Department of Automation and Control Engineering. \\ Avenida Nereu Ramos, 3450-D Chapecó/SC. \\ ${ }^{4}$ Faculdade Empresarial de Chapecó (UCEFF) - Department of Environmental and Sanitary Engineering. \\ Rua Lauro Muller, 767 E, Chapecó/SC.
}

\begin{abstract}
This paper presents the mathematical modeling of wind turbines that do not use conventional blades capture the kinetic energy of the winds. In place of the blades, the turbine has rotating cylinder driven by one or more electric motors. Rotating cylinders immersed in air create differences pressure leading forces (support) transverse to the direction of air flow. This physical phenomenon is known as the Magnus Effect. These forces applied to a turbine rotating cylinders provide the torque on the main shaft that is transmitted to the electric generator. We present a mathematical model of conventional wind turbines and a performs comparative in terms of mechanical power with wind turbine Magnus.
\end{abstract}

Keywords: Wind turbine, Magnus Effect, Rotating cylinder, Magnus turbine. 